\title{
Perceived numerosity as a function of array number, speed of array development, and density of array items
}

\author{
WALTER H. HOLLINGSWORTH, J. PAUL SIMMONS, TAMMY R. COATES, \\ and HENRY A. CROSS \\ Mobile College, Mobile, Alabama
}

\begin{abstract}
College-student subjects engaged in judgments of numerosity received stimulus arrays on a computer screen that varied in their number level (low, medium, and high), the speed with which the array developed (slow, medium, or fast), and the density of the items in the array (low or high density). The subjects overestimated number when the array was small (75-125 items) but significantly underestimated number when it was at a medium (150-250) or high level (550-650). Density of array items was a significant variable, since subjects showed more underestimation under the high-density condition. Additionally, there was a speed $x$ density interaction. The results are discussed with respect to which class of variable, sensory or cognitive, most influences perceived numerosity.
\end{abstract}

Although they deal with the animal literature, Davis and Pérusse (1988), in their excellent review, distinguish among a number of approaches to the measurement of numerical competence. One of the approaches, estimation, is different from subitizing because the subject is required to assign a numerical tag, without counting, to a relatively large number of items (Kaufman, Lord, Reese, \& Volkman, 1949).

A host of studies on numerosity perception have employed magnitude estimation (e.g., Kemp, 1984; Kowal, 1987; Krueger, 1982, 1984; Masin, 1983), but others (e.g., Cousins \& Ginsburg, 1983; Ginsburg, 1980; Ginsburg \& Nicholls, 1988; Ginsburg \& Riedel, 1983) have relied on a simple estimation procedure. In the second category of studies, the usual procedure has been to present various dot arrays briefly and to require a verbal estimate on the termination of the array. Such studies have shown a tendency for subjects to underestimate the numerical size of the array, a finding that is in harmony with the magnitude-estimation studies (e.g., Kemp, 1984; Krueger, 1982). To summarize both sets of investigations, the underestimation finding has generally been shown to increase with the magnitude of the array and to be more pronounced among females (Krueger, 1982). However, if females self-select into such a study, the gender factor may not be significant (Krueger, 1984). Furthermore, the relative underestimation has been shown to be reduced in magnitude for the following: type of physical background (Kemp, 1984), melodic familiarity when number of notes was judged (Kowal, 1987), regularity or organi-

Henry A. Cross is Emeritus Professor of Psychology, Colorado State University, Ft. Collins, CO. Correspondence should be sent to him during the 1991-1992 academic year at the Department of Psychology, St. Mary's College of Maryland, St. Mary's City, MD 20686. zation ("patterning") of the stimulus array (Ginsburg, $1976,1978)$, a smaller size of the items constituting the array (Ginsburg \& Nicholls, 1988), training (Kemp, 1984), and feedback (Krueger, 1982).

Krueger (1984) has addressed the issue of the relative contribution of "cognitive" versus "sensory and/or perceptual" factors in the ability to estimate numerosity. By sensory or perceptual factors, he seems to point to the early processing stages of various physical aspects of the array, such as the discriminability of items, their organization, and their size, which some of the above studies have implicated. Cognitive factors would include the more "interpretative" aspects of processing, such as those involved in the type of instructions and feedback one receives.

The present study was designed to examine further some possible sensory/perceptual variables in numerosity perception, namely, those relating to the speed with which an array develops and to the stimulus density of the items of the array. Low-, medium-, and high-number arrays were presented on a computer screen under three speeds of array development (slow, medium, or fast) and two levels of stimulus-item density (low or high).

\section{METHOD}

\section{Subjects}

Nine volunteer subjects, 8 females and 1 male, from a general psychology class received special credit for participating.

\footnotetext{
Apparatus

Stimuli were presented on an IBM PC/XT compatible computer set to run at the standard operating speed of $4.77 \mathrm{MHz}$. A BASIC program generated three levels of random asterisks on the monitor: low (75-125), medium (150-250), and high (550-650). The particular number on a given trial was randomly generated. Asterisks appeared on the screen in one of three speeds: slow, medium, and fast. Speed was controlled by creating a delay between individual asterisks with a loop that varied from 0 to $.22 \mathrm{sec}$. The medium speed delay was $.05 \mathrm{sec}$. Two density
} 
levels were generated by using either a full screen (low) or a half screen (high density). The low density utilized a total of 1,920 ( 80 columns $\times 24$ rows) positions, whereas the high density condition employed half of the screen, or a possible $960(40 \times 24)$ points. In all cases, the completed matrix remained on the screen for $1.5 \mathrm{sec}$, after which the screen was cleared and the subjects typed in their number estimation. The touch of any key then activated the start of the next display.

\section{Design and Procedure}

Each subject received nine trials (three per week) over a 3-week period. A given trial consisted of 18 conditions that were factorial combinations of three number levels (low, medium, and high), three speeds (slow, medium, and fast), and two density levels (low and high). Thus, for each subject, there were three scores in each of the 18 conditions for each week. The 18 conditions within a given trial were randomized separately for each subject.

The subjects were instructed to start the display and to type in their number estimate when the display disappeared. They were asked not to try to count but to simply relax during the presentation and then make their best estimate as to the number of "dots" for each succeeding display. No feedback was provided on any trial.

\section{RESULTS}

The computer program recorded the subject's response and gave the percentage of error for each judgment, and, since there were three weekly trials for each condition, both means and medians were computed on each of the 18 conditions for each week. Since these data were analyzed over 3 weeks, each subject had 54 total scores in a four-factor completely within-subjects analysis-ofvariance design.

One female subject had scores in line with the others during Week 1 , but during Weeks 2 and 3 , her scores became aberrant such that her estimations hovered around a $500 \%$ overestimation. Her data were not included in the analysis because of their extreme deviation and because of her claim, when questioned, that she had "discovered the system."

Mean-percentage-of-error scores were analyzed and revealed a significant number effect $[F(2,14)=30.56$, $p<.00001$ ]. The low number was overestimated by a mean of $8.24 \%$, whereas the medium and high numbers were underestimated with means of -13.26 and -27.63 , respectively. Subsequent post hoc tests using the NeumanKeuls procedure indicated that all mean comparisons were significant $(\alpha=.01)$.

The density factor also yielded a significant $F$ ratio $[(1,7)=9.47, p<.02]$, with the low-density condition resulting in a mean underestimation of $-1.00 \%$ compared with $-20.77 \%$ for the high-density condition. No other factors reached significance, but speed approached significance $[F(2,14)=3.02, p<.08]$, as did weeks $\times$ density $[F(2,14)=3.20, p<.07]$ and weeks $\times$ speed $[F(4,28)=2.24, p<.09]$.

Median-percentage-of-error scores were also analyzed and gave almost parallel results. Number was again significant $[F(2,14)=23.56, p<.00001]$. The median percentage of error for high, medium, and low numbers were $-27.38,-14.31$, and 6.26 , respectively. Subsequent

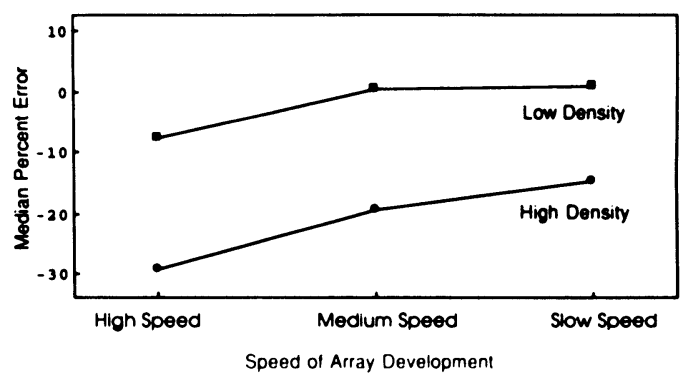

Figure 1. Median percentage of error showing the density effect and the density $x$ speed interaction.

post hoc tests with the Neuman-Keuls revealed that although the high and the medium number produced errors that were not different, both showed significantly greater error than did the low number group $(\alpha=.01)$. Median percentage error attributable to density was significant $[F(1,7)=8.92, p<.02]$, with the high-density condition producing more error $(-21.29)$ than the lowdensity condition $(-2.33)$. In the analysis involving median percentage error, the speed factor approached significance $[F(2,14)=3.28, p<.07]$, and there was a speed $\times$ density interaction $[F(2,14)=4.44, p<.03]$. Figure 1 depicts these findings. It is apparent that the highdensity condition results in a greater underestimation than does the low-density condition and that this underestimation is reduced as one is moved from high speed through medium speed to low speed. The interaction can be observed in the fact that the difference in the density conditions decreases as one moves from high to low speed.

\section{DISCUSSION}

The observed systematic underestimation is consistent with other studies, although it was not acutely manifest with the low-density condition except under the high-number treatment. Additionally, the overall overestimation for the low-number condition is not the typical finding, although it has sometimes been observed when number size is quite small.

Of interest is the finding that array density, which must obviously be interpreted as a sensory or perceptual factor, as opposed to a cognitive one, is an important factor in perceived numerosity. A dense array results in smaller estimates and underestimation in the medium- and highnumber conditions than does a more diffuse one.

The speed with which an array develops, although not statistically reliable, did approach significance for both the means and medians analyses, and it is possible that extending the range of speeds would result in a significant finding. Additionally, the fact that the density $\times$ speed interaction approached significance in the means analysis and was, in fact, significant in the medians analysis further implicates the speed factor and points to the need for additional investigation of both speed and density.

Although it may well be true that cognitive factors are more important in perceived numerosity than are sensory and/or perceptual ones (Krueger, 1984), the present findings suggest that we may not yet have identified all the sensory/perceptual factors of importance. For future research, it would be useful to manipulate some of the important cognitive factors, for example, instructions and feedback, in conjunction with the variables of this experiment. 


\section{REFERENCES}

Cousins, J. B., \& Ginsburg, N. (1983). Subjective correlation and the regular-random numerosity illusion. Journal of General Psychology, 108, 3-10.

DAvis, H., \& Pérusse, R. (1988). Numerical competence in animals: Definitional issues, current evidence, and a new research agenda. Behavioral \& Brain Sciences, 11, 561-615.

GiNSBURG, N. (1976). Effect of item arrangement on perceived numerosity: Randomness vs. regularity. Perceptual \& Motor Skills, 43, 663-668.

GinsBurg, N. (1978). Perceived numerosity, item arrangement, and expectancy. American Journal of Psychology, 91, 267-273.

GinsBurg, N. (1980). The regular-random illusion: Rectangular patterns. Journal of General Psychology, 103, 211-216.

GinsBurg, N., Nicholls, A. (1988). Perceived numerosity as a function of item size. Perceptual \& Motor Skills, 67, 656-658.

GinsBurg, N., \& RIEDEL, H. (1983). Numerosity estimation with successive presentation: Item organization. Journal of General Psychology, 108, 169-173.
Kaufman, E., Lord, M., Reese, T., \& Volkman, J. (1949). The discrimination of visual number. American Journal of Psychology, 62 , 498-525.

KeMP, S. (1984). Estimating the sizes of sports crowds. Perceptual \& Motor Skills, 59, 723-729.

Kowal, K. H. (1987). Apparent duration and numerosity as a function of melodic familiarity. Perception \& Psychophysics, 42, 122-131.

Krueger, L. E. (1982). Single judgments of numerosity. Perception \& Psychophysics, 31, 175-182.

KRUEger, L. E. (1984). Perceived numerosity: A comparison of magnitude production, magnitude estimation, and discrimination judgments. Perception \& Psychophysics, 35, 536-542.

Masin, S. (1983). Null effect of intramodal stimulus-range variation on the exponent for numerousness. Perceptual \& Motor Skills, 56, 851-855. 